\title{
A Note on the Computation of Multiplicities
}

\author{
Eduard Boda Peter Schenzel * \\ Comenius University, Department of Geometry \\ 84248 Bratislava, Slovakia \\ e-mail: eduard.boda@fmph.uniba.sk \\ Martin-Luther-Universität Halle-Wittenberg, Fachbereich Mathematik und Informatik \\ D - 06099 Halle (Saale), Germany \\ e-mail: schenzel@mathematik.uni-halle.de
}

MSC 2000: 13H15 (primary); 13B02 (secondary)

Keywords: multiplicity, finite extension, additivity formula

\section{Introduction}

Let $f_{1}, \ldots, f_{r}$ denote a system of polynomials in the polynomial ring $P=k\left[x_{1}, \ldots, x_{d}\right]$ such that

$$
\underline{0}=(0, \ldots, 0) \in V\left(f_{1}, \ldots, f_{r}\right) \subset \mathbb{A}_{K}^{n} .
$$

Suppose that $f R$ is an $\underline{x} R$-primary ideal, where $R=P_{(\underline{x})}$. Then the Hilbert-Samuel multiplicity $e_{0}(f ; R)$ provides a certain information about the local structure of the affine variety $V=V\left(f_{1}, \ldots, f_{r}\right)$ as considered in Bézout's theorem and related problems.

The goal of our interest in this note is the following question: Suppose that a $d$-dimensional local Noetherian ring $(A, \mathfrak{m}, k)$ contains the residue field $k$. Let $\underline{x}=x_{1}, \ldots, x_{d}$ denote a system of parameters of $A$. Suppose that the polynomials $f$ satisfy the above requirements. What is the relation between the multiplicities $e_{0}(\underline{f} ; R)$ and $e_{0}(\underline{f} ; A)$ ? Recall that the system of parameters $\underline{x}$ in $A$ is algebraically independent (cf. [2, Corollary 11.21]), so that $P=$ $k\left[x_{1}, \ldots, x_{d}\right]$ is a polynomial subring of $A$.

*The authors are grateful to the DAAD and the Slovak Ministry of Education (Grant Nr. 1/7658/20) for supporting the research concerning this article.

0138-4821/93 \$2.50 (c) 2004 Heldermann Verlag 
Theorem 1.1. Let $(A, \mathfrak{m}, k)$ denote a d-dimensional local ring containing the residue field $k$. Suppose that $f R$ is an $\underline{x} R$-primary ideal. Let $\underline{x}=x_{1}, \ldots, x_{d}$ denote a system of parameters of $A$. Then

$$
e_{0}(\underline{f} ; A)=e_{0}(\underline{f} ; R) e_{0}(\underline{x} ; A),
$$

where $R=k[\underline{x}]_{(\underline{x})}$ denotes the localized polynomial ring.

We will prove this result in Section 3 as a consequence of the additivity formula for multiplicities. This formula as well as further preliminaries are summarized in Section 2. In fact, we discuss the behavior of the multiplicity with respect to a short exact sequence.

As an application there is the following Cohen-Macaulay criterion for the rings we consider here.

Corollary 1.2. With the previous notation we have the following inequality

$$
e_{0}(\underline{f} ; A) \leq e_{0}(\underline{f} ; R) L_{A}(A / \underline{x} A) .
$$

The equality holds if and only if $A$ is a Cohen-Macaulay ring.

We continue the considerations with an example for the computation of multiplicities motivated by the work of [3]. Furthermore we conclude with a short, direct proof of Serre's formula for the multiplicity of a system of parameters. A similar to Serre's approach was developed by Auslander and Buchsbaum (cf. [1]).

Corollary 1.3. With the previous assumptions let $\underline{x}=x_{1}, \ldots, x_{n}$ denote a system of parameters of a finitely generated A-module $M$. Then

$$
e_{0}(\underline{x} ; M)=\sum_{i=0}^{n}(-1)^{i} L_{A}\left(H_{i}(\underline{x} ; M)\right),
$$

where $H_{i}(\underline{x} ; M), i \geq 0$, denotes the Koszul homology of $M$ with respect to $\underline{x}$.

See the third section for all the definitions. Of course the result is true without the assumption that $(A, \mathfrak{m}, k)$ contains $k$ (cf. [6] resp. [1]). We present here a proof without the use of spectral sequences as a consequence of the additivity formula. Moreover it could be of some interest for a short, direct approach to the multiplicity theory.

\section{On the additivity formula}

In the following let $(A, \mathfrak{m})$ denote a local Noetherian ring. Let $M$ be a finitely generated $A$-module. We say that an ideal $I$ of $A$ is an ideal of definition with respect to $M$ provided the $A$-module $M / I M$ is of finite length.

Then it follows that $M / I^{n} M$ is an $A$-module of finite length for all $n \in \mathbb{N}$. For large $n$ this length function becomes a polynomial in $n$ usually written as

$$
L_{A}\left(M / I^{n} M\right)=\sum_{i=0}^{d} e_{i}(I ; M)\left(\begin{array}{c}
n+d-i-1 \\
d-i
\end{array}\right),
$$


where its degree $d$ coincides with $\operatorname{dim}_{A} M$, the dimension of $M$ (cf. e.g. [5, p. 107]).

We call the integer coefficients $e_{i}(I ; M)$ the Hilbert-Samuel multiplicities of $M$ with respect to $I$. The first of it, $e_{0}(I ; M)$, is just called the multiplicity of $M$ with respect to $I$. It covers various interesting information concerning the properties of $M$, in particular for parameter ideals with respect to $M$.

Lemma 2.1. With the previous notation let $0 \rightarrow M^{\prime} \rightarrow M \rightarrow M^{\prime \prime} \rightarrow 0$ denote a short exact sequence of $A$-modules. Let I denote an ideal of $A$.

a) The ideal $I$ is an ideal of definition of $M$ if and only if it is one for both $M^{\prime}$ and $M^{\prime \prime}$.

b) Suppose that I satisfies the equivalent conditions in a). Then

$$
L_{A}\left(M^{\prime} / I^{n} M^{\prime}\right)+L_{A}\left(M^{\prime \prime} / I^{n} M^{\prime \prime}\right)-L_{A}\left(M / I^{n} M\right)
$$

is for large $n$ a polynomial of degree $\leq \operatorname{dim} M^{\prime}-1$.

Proof. The given short exact sequence of $A$-modules induces an exact sequence

$$
0 \rightarrow I^{n} M \cap M^{\prime} / I^{n} M^{\prime} \rightarrow M^{\prime} / I^{n} M^{\prime} \rightarrow M / I^{n} M \rightarrow M^{\prime \prime} / I^{n} M^{\prime \prime} \rightarrow 0
$$

for any integer $n \geq 1$. Now let $I$ be an ideal of definition for $M^{\prime}$ and $M^{\prime \prime}$. Then the sequence considered for $n=1$ provides the finite length of $M / I M$. Therefore $I$ is also an ideal of definition for the $A$-module $M$.

Now let $I$ be an ideal of definition for $M$. Then it will be immediately also an ideal of definition for $M^{\prime \prime}$. Moreover, by the Artin-Rees lemma (cf. [5, Theorem 8.5]) there is an integer $k$ such that

$$
I^{n+k} M \cap M^{\prime} \subseteq I^{n} M^{\prime} \text { for all } n \geq 1 .
$$

Now consider the corresponding injective homomorphism of $A$-modules

$$
0 \rightarrow M^{\prime} / I^{k+1} M \cap M^{\prime} \rightarrow M / I^{k+1} M .
$$

It provides that $M^{\prime} / I^{k+1} M \cap M^{\prime}$ - and also $M^{\prime} / I M^{\prime}$ as an epimorphic image - is an $A$-module of finite length. So $I$ is an ideal of definition for $M^{\prime}$.

In order to prove b) first note that the above exact sequence provides the following equality for the length functions

$$
L_{A}\left(M^{\prime} / I^{n} M^{\prime}\right)+L_{A}\left(M^{\prime \prime} / I^{n} M^{\prime \prime}\right)-L_{A}\left(M / I^{n} M\right)=L_{A}\left(I^{n} M \cap M^{\prime} / I^{n} M^{\prime}\right)
$$

for all $n \geq 1$. That means, for large $n$ the length function at the left hand side is a polynomial in $n$. In order to finish we have to estimate its degree. To this note that

$$
L_{A}\left(I^{n+k} M \cap M^{\prime} / I^{n+k} M^{\prime}\right) \leq L_{A}\left(I^{n} M^{\prime} / I^{n+k} M^{\prime}\right)
$$

for all $n \geq 1$ as follows by the Artin-Rees lemma above. But now we know that $L_{A}\left(I^{n} M^{\prime} /\right.$ $I^{n+1} M^{\prime}$ ) is for large $n$ a polynomial of degree $\operatorname{dim}_{A} M^{\prime}-1$. By summarizing $k-1$ subsequent polynomials for a large $n$ provides the estimate of the claim stated in b).

The previous is a slight simplification of an argument by Flenner and Vogel (cf. [4]). It applies to the computation of multiplicities concerning modules that are related by a short exact sequence. That is, there is the following additivity formula. 
Corollary 2.2. Let $0 \rightarrow M^{\prime} \rightarrow M \rightarrow M^{\prime \prime} \rightarrow 0$ be a short exact sequence of $A$-modules. Suppose that $I$ is an ideal of definition for $M$. Then

$$
e_{0}(I ; M)=e_{0}\left(I ; M^{\prime}\right)+e_{0}\left(I ; M^{\prime \prime}\right) .
$$

Moreover $e_{0}(I ; M)=e_{0}\left(I ; M^{\prime}\right)$ resp. $=e_{0}\left(I ; M^{\prime \prime}\right)$ provided $\operatorname{dim} M^{\prime \prime}<\operatorname{dim} M$ resp. $\operatorname{dim} M^{\prime}<$ $\operatorname{dim} M$.

Proof. The proof of the multiplicity formula is a consequence of 2.2. It follows by comparing the coefficients of degree $d=\operatorname{dim} M$ of the corresponding Hilbert functions. Note that under the additional dimension inequalities the corresponding leading coefficient of the Hilbert polynomial will be zero.

With these preliminary results we are prepared to prove the additivity formula for multiplicities. The result is well-known. We have included here a proof as a consequence of 2.1 which we believe is of some interest in itself.

For an $A$-module $M$ we denote by $\operatorname{Assh}_{A} M=\left\{\mathfrak{p} \in \operatorname{Ass}_{A} M \mid \operatorname{dim} A / \mathfrak{p}=\operatorname{dim} M\right\}$ the set of highest dimensional associated prime ideals of $M$.

Lemma 2.3. Let $M$ denote a finitely generated A-module. Suppose that $I$ is an ideal of definition of $M$. Then

$$
e_{0}(I ; M)=\sum_{\mathfrak{p} \in \operatorname{Assh} M} e_{0}(I ; A / \mathfrak{p}) L_{A_{\mathfrak{p}}}\left(M_{\mathfrak{p}}\right)
$$

Proof. By view of $\left[5\right.$, Theorem 6.4] there is a finite filtration $\left\{M_{i}\right\}_{0 \leq i \leq t}$ such that

$$
0 \subset M_{t} \subset \ldots \subset M_{1} \subset M_{0}=M \text { and } M_{i-1} / M_{i} \simeq A / \mathfrak{p}_{i}
$$

where $\mathfrak{p}_{i}, i=1, \ldots, t$ denotes a prime ideal in $A$. Moreover

$$
\operatorname{Ass}_{A} M \subseteq\left\{\mathfrak{p}_{1}, \ldots, \mathfrak{p}_{t}\right\} \subseteq \operatorname{Supp}_{A} M
$$

and all of the three sets have the same set of minimal elements. Therefore

$$
e_{0}(I ; M)=\sum_{i=1}^{t} e_{0}\left(I ; A / \mathfrak{p}_{i}\right),
$$

as follows by 2.2. Clearly $e_{0}\left(I ; A / \mathfrak{p}_{i}\right)=0$ whenever $\operatorname{dim} A / \mathfrak{p}_{i}<\operatorname{dim} M$. So it will be enough to take the sum over all primes in Assh $M$. In order to finish the proof note that $e_{0}(I ; A / \mathfrak{p})$ occurs as many times as $L_{A_{\mathfrak{p}}}\left(M_{\mathfrak{p}}\right)$. This follows by an easy localization argument.

\section{On a formula for multiplicities}

Now let $f_{1}, \ldots, f_{r}$ denote a system of polynomials in the polynomial ring $P=k\left[x_{1}, \ldots, x_{d}\right]$ such that

$$
\underline{0}=(0, \ldots, 0) \in V\left(f_{1}, \ldots, f_{r}\right) \subset \mathbb{A}_{K}^{n} .
$$


Suppose that $f R$ is an $\underline{x} R$-primary ideal, where $R=P_{(\underline{x})}$. Then the Hilbert-Samuel multiplicity $e_{0}(\underline{f} ; \bar{R}), R=P_{(\underline{x})}$ provides a certain information about the local structure of the affine variety $V=V\left(f_{1}, \ldots, f_{r}\right)$ as considered in Bézout's theorem and related problems.

Let $(A, \mathfrak{m}, k)$ denote a local ring containing the residue field $k$. Let $\underline{x}=x_{1}, \ldots, x_{d}$ denote a system of parameters of $A$. Then $k[\underline{x}]$ is a polynomial subring of $A$ (cf. [2, Corollary 11.21]) such that $A$ is a finitely generated $k[\underline{x}]$-module. It induces an embedding of $R=k[\underline{x}]_{(\underline{x})}$ into $A$ such that $A$ is a finitely generated $R$-module.

Theorem 3.1. With the previous notation we have

$$
e_{0}(\underline{f} ; A)=e_{0}(\underline{f} ; R) e_{0}(\underline{x} ; A) .
$$

Proof. By our construction the fibre ring $A / \underline{x} A$ of the local homomorphism

$$
(R,(\underline{x})) \rightarrow(A, \mathfrak{m})
$$

is of dimension zero. Therefore $\mathfrak{m}^{n} \subseteq \underline{x} A$ for a certain integer $n \in \mathbb{N}$. Moreover $\underline{x}^{k} R \subseteq \underline{f} R \subseteq$ $\underline{x} R$ for a certain integer $k \in \mathbb{N}$. This implies the following containment relation

$$
\mathfrak{m}^{n k} \subseteq \underline{x}^{k} A \subseteq \underline{f} A \subseteq \underline{x} A \subseteq \mathfrak{m} .
$$

Therefore $f A$ is an $\mathfrak{m}$-primary ideal.

Now the additivity formula applied to the multiplicity $e_{0}(\underline{f} ; A)$ provides the following equality

$$
e_{0}(\underline{f} ; A)=e_{0}(\underline{f} ; R) L_{R_{(0)}}\left(A_{(0)}\right) .
$$

Recall that $A$ is a finitely generated $R$-module. Moreover, $R$ is a domain with the single minimal prime (0).

In order to finish the proof we have to show that the rank of $A$ over $R$, i.e. $L_{R_{(0)}}\left(A_{(0)}\right)$ is equal to the multiplicity $e_{0}(\underline{x} ; A)$. In fact, this is another consequence of the additivity formula. More precisely it follows that

$$
e_{0}(\underline{x} ; A)=e_{0}(\underline{x} ; R) L_{R_{(0)}}\left(A_{(0)}\right) .
$$

But now $e_{0}(\underline{x} ; R)=1$, because $(R,(\underline{x}))$ is a regular local ring.

In fact, the previous result 3.1 proves the claim of the result 1.1 of the introduction. Now we continue with the corollary about the Cohen-Macaulayness of $(A, \mathfrak{m})$.

Corollary 3.2. With the previous notation we have the following inequality

$$
e_{0}(\underline{f} ; A) \leq e_{0}(\underline{f} ; R) L_{A}(A / \underline{x} A) .
$$

The equality holds if and only if $A$ is a Cohen-Macaulay ring.

Proof. Let $\underline{x}=x_{1}, \ldots x_{d}$ denote a system of parameters of $A$. Then it is well known that

$$
e_{0}(\underline{x} ; A) \leq L_{A}(A / \underline{x} A) .
$$

Moreover equality holds if and only if $A$ is a Cohen-Macaulay ring (cf. [5, Theorem 17.11]. So the claim of the corollory is a consequence of 3.1.

This proves the corollary 1.2 of our Introduction. 
Remark 3.3. By the Auslander-Buchsbaum formula it follows that $A$ in 3.2 is CohenMacaulay if and only if it is free as an $R$-module. Let us contribute with another argument by the aid of multiplicities. By the Nakayama lemma the minimal number of generators of $A$ as $R$-module is given by

$$
\operatorname{dim}_{R / \underline{x} R} A / \underline{x} A=L_{A}(A / \underline{x} A)=: e .
$$

Then there is a short exact sequence of $R$-modules

$$
0 \rightarrow C \rightarrow R^{e} \rightarrow A \rightarrow 0,
$$

where $C$ denotes the kernel of the natural surjection. Since $A$ is Cohen-Macaulay the sequence $\underline{x}$ is $A$-regular. So it induces a short exact sequence

$$
0 \rightarrow C / \underline{x} C \rightarrow(R / \underline{x} R)^{e} \rightarrow A / \underline{x} A \rightarrow 0 .
$$

By counting the length it implies $C / \underline{x} C=0$. Therefore $C=0$ by Nakayama's Lemma. That is, $A$ is a free $R$-module.

Finally we illustrate the previous results by an example considered in [3].

Example 3.4. Let $(A, \mathfrak{m}, k)$ denote a two dimensional local ring containing the residue field $k$. Suppose that $\underline{x}=a, b$ denotes a system of parameters of $A$. Then there are among others the following computations for multiplicities (cf.[3])

a) $e_{0}\left(a^{m}-b^{n}, a^{k}-b^{l} ; A\right)=e_{0}(\underline{x} ; A) \min \{m l, n k\}$, provided $m l \neq n k$.

b) $e_{0}\left(a^{m}, b^{n}, a^{k} b^{l} ; A\right)=e_{0}(\underline{x} ; A) \min \{m n, m l+n k\}$.

Moreover, let $A=k\left[\left[s^{4}, s^{3} t, s t^{3}, t^{4}\right]\right]$, where $s, t$ denote two variables over $k$. Then $\underline{x}=a, b$ with $a=s^{4}, b=t^{4}$ forms a system of parameters of $A$. By b) it follows that

$$
e_{0}\left(a^{m}, b^{n}, a^{k} b^{l} ; A\right)=4 \cdot \min \{m n, m l+n k\} .
$$

Note that $e_{0}(\underline{x} ; A)=4$ while $L_{A}(A / \underline{x} A)=5$ as it is easily seen. We remind that $A$ is not a Cohen-Macaulay ring.

\section{On Serre's multiplicity formula}

In the following let $(A, \mathfrak{m})$ denote a local ring. Let $M$ be a finitely generated $A$-module. For the investigations in this section let $\underline{x}=x_{1}, \ldots, x_{n}$ denote a system of elements of the maximal ideal $\mathfrak{m}$ of $A$ such that $L_{A}(M / \underline{x} M)$ is finite.

Let $K_{\bullet}(\underline{x} ; M)$ denote the Koszul complex of $\underline{x}$ with respect to $M$ (cf. [6, Chapitre 4] resp. [1]). It follows (cf. [6]) that the length of the Koszul homology modules $H_{i}(\underline{x} ; M)$ is finite for all $i=0,1, \ldots, n$. Moreover it follows by the construction that $H_{i}(\underline{x} ; M)$ vanishes for all $i<0$ and $i n$.

Definition 4.1. With the previous notation let us define

$$
\chi_{A}(\underline{x} ; M)=\sum_{i \in \mathbb{Z}}(-1)^{i} L_{A}\left(H_{i}(\underline{x} ; M)\right)
$$

the Euler characteristic of $\underline{x}$ with respect to $M$. 
Recall that $\chi_{A}(\underline{x} ; M)$ is a well defined integer. In the following result we will relate it to the multiplicity symbol $e_{0}(\underline{x} ; M)$.

Theorem 4.2. Suppose that $(A, \mathfrak{m}, k)$ contains the residue field $k$. With the previous notation and definition it follows that

$$
\chi_{A}(\underline{x} ; M)=e_{0}(\underline{x} ; M)
$$

provided $n=\operatorname{dim}_{A} M$, i.e. $\underline{x}=x_{1}, \ldots, x_{n}$ is a system of parameters of $M$.

Proof. First of all we may pass to the $\operatorname{ring} A / \operatorname{Ann}_{A} M$, so that $\operatorname{dim}_{A} M=n$ without loss of generality. As in the third section let $R=k[\underline{x}]_{(\underline{x})}$ denote the regular local ring contained in $A$. As in the proof of Theorem 3.1 it follows that $e_{0}(\underline{x} ; M)=L_{R_{(0)}}\left(M_{(0)}\right)$ as an easy consequence of the additivity formula.

Next recall that $M$ - considered as an $R$-module - is finitely generated. Clearly as an $R$-module it is of finite projective dimension. But now $(\underline{x})$ generates the maximal ideal of the regular local ring $R$. Therefore there are the following isomorphisms

$$
\operatorname{Tor}_{i}^{R}(k, M) \simeq H_{i}(\underline{x} ; M), i \in \mathbb{Z} .
$$

Let $0 \rightarrow F_{n} \rightarrow \ldots \rightarrow F_{1} \rightarrow F_{0} \rightarrow M \rightarrow 0$ denote a minimal free resolution of $M$ over $R$. Recall that $M$ is of finite projective dimension as an $R$-module. But now

$$
\operatorname{rank} F_{i}=\operatorname{dim} \operatorname{Tor}_{i}^{R}(k, M)
$$

for all $i \in \mathbb{Z}$. Furthermore the rank of $M$ is given by

$$
\operatorname{rank} M=L_{R_{(0)}}\left(M_{(0)}\right)=e_{0}(\underline{x} ; M)
$$

as follows by the additivity formula (cf. 2.3). By counting all the ranks in the minimal free resolution of $M$ it provides that

$$
\operatorname{rank} M=\sum_{i \in \mathbb{N}}(-1)^{i} \operatorname{rank} F_{i} .
$$

By using the above isomorphisms the claim is shown to be true. Note that the length is the same over $R$ and over $A$.

Of course this result is true without any additional assumption on the local ring $(A, \mathfrak{m}, k)$ (cf. [6, Chapitre 4] and [1]). We have here included this short, direct argument (without a spectral sequence) as another application of the additivity formula. In fact it proves 1.2 of the introduction.

\section{References}

[1] Auslander, M.; Buchsbaum, D. A.: Codimension and Multiplicity. Ann. of Math. 68 (1958), 625-657.

Zbl 0092.03902

[2] Atiyah, M. F., MacDonald, I. G.: Introduction to Commutative Algebra. Addison Wesley, Massachusetts 1969. 
[3] Boda, E.; Solčan, S.: On the multiplicity of $\left(x_{1}^{m}, x_{2}^{n}, x_{1}^{k} x_{2}^{l}\right)$. Acta Math. Univ. Comenianae LII-LIII (1987), 297-299.

Zbl 0669.13011

[4] Flenner, H.; Vogel, W.: On multiplicities of local rings. Manuscr. Math. 78 (1993), 85-97.

Zbl 0794.13016

[5] Matsumura, H.: Commutative Ring Theory. Cambridge Univ. Press, 1986.

Zbl 0603.13001

[6] Serre, J.-P.: Algèbre Locale - Multiplicités. Lect. Notes in Math., Vol. 11, Trois. Édt., Springer, 1975.

Zbl 0296.13018

Received February 18, 2003 